\title{
AVALIAÇÃO DO PERFIL SENSORIAL DE ALIMENTO COM SOJA SABOR LARANJA
}

\author{
RENATA TORREZAN * \\ CAROLINA MARTINEZ CECCATO * \\ ANDRÉA CARLA DA SILVA BARRETTO * \\ VALQUÍRIA SEIXAS DA SILVA * \\ CARLA CARATIN * \\ CAMILA GAMBINI PEREIRA * \\ JULIAN MARTINEZ * \\ MARTA MITSUI KUSHIDA * \\ MANUEL PINTO NETO * \\ BEATRIZ IAMANAKA * \\ HELENA MARIA ANDRÉ BELLINI CARDELLO **
}

Três produtos comerciais de alimento com soja sabor laranja (A, B e C), acondicionados em embalagem longa vida, adquiridos no mercado foram avaliados sensorialmente. A Análise Descritiva Quantitativa (ADQ) foi utilizada para traçar os perfis sensoriais, avaliando-se doze atributos levantados por 10 julgadores selecionados e treinados. A aceitação dos produtos foi avaliada por 30 potenciais consumidores mediante Teste Afetivo, realizado em laboratório, utilizando-se escala hedônica estruturada mista de 9 pontos. Os resultados da ADQ foram submetidos à Análise de Variância (ANOVA), Teste de Média de Tukey e Análise de Componentes Principais, já os resultados do Teste Afetivo (Aceitação) foram analisados pela ANOVA e pelo Teste de Média de Tukey. Os produtos A e B ficaram mais próximos entre si, o produto C diferiu do produto B (significativamente ao nível de 5\%) em todos os atributos, com exceção da doçura e amargor. Os produtos $\mathrm{A}$ e $\mathrm{C}$ diferiram em todos os atributos, com exceção do residual de edulcorante e viscosidade. No teste afetivo, o produto A obteve as maiores médias e notas dos julgadores, não diferindo do produto $B$ em nenhum dos atributos analisados.

PALAVRAS-CHAVE: ALIMENTO COM SOJA - ANÁLISE SENSORIAL; ANÁLISE DESCRITIVA QUANTITATIVA; TESTE AFETIVO.

* Alunos do Curso de Pós-graduação da Faculdade de Engenharia de Alimentos (FEA), Universidade Estadual de Campinas (UNICAMP), Campinas, SP - Brasil (email: torrezan@fea.unicamp.br).

** Professora Doutora em Tecnologia de Alimentos, Laboratório de Análise Sensorial, Departamento de Alimentos e Nutrição, FEA, UNICAMP, Campinas, SP - Brasil (email: hellini@fea.unicamp.br). 


\section{INTRODUÇÃO}

O Brasil é o segundo produtor mundial de soja, cujo complexo agroindustrial movimenta anualmente cerca de US\$50 bilhões (NOTÍCIAS..., 2004). No entanto, o consumo humano dessa leguminosa e de seus derivados é ainda restrito no Brasil em razão de aspectos socioculturais e hábitos alimentares da população.

O mercado de bebidas não-alcoólicas brasileiro está em plena expansão, particularmente o de suco de frutas (BEBIDAS..., 2001). Os sucos de fruta acrescidos de produtos de soja apresentam enorme potencial de crescimento, pois conciliam as características sensoriais desejáveis das frutas com as propriedades funcionais da soja (ALIMENTOS..., 2000). Segundo a KRONES (2003), o crescimento em volume de vendas de bebidas à base de proteína de soja foi de 104\% no primeiro semestre de 2002.

A análise sensorial é essencial para medir e interpretar as reações produzidas pelas características dos alimentos e a forma como são percebidas pelos sentidos humanos (ABNT, 1993). Os testes sensoriais podem ser divididos em métodos discriminativos ou de diferença, descritivos ou analíticos, e afetivos (MEILGAARD, CIVILLE e CARR, 1999). Os descritivos avaliam a intensidade dos atributos sensoriais dos produtos (aspectos quantitativos) e descrevem o produto avaliado (aspecto qualitativo). Os métodos descritivos mais conhecidos são o Perfil de Sabor (MEILGAARD, CIVILLE e CARR, 1999), a Análise Descritiva Quantitativa (STONE e SIDEL, 1993) e o Perfil de Textura (CIVILLE e SZCESNIAK, 1973).

A Análise Descritiva Quantitativa (ADQ) avalia os aspectos de todos os atributos do produto (aparência, aroma, sabor e textura). Uma vez selecionados os julgadores, a primeira etapa a ser cumprida é o desenvolvimento da terminologia descritiva. O treinamento e a seleção definitiva dos julgadores são realizados com os próprios produtos e materiais de referência, previamente estabelecidos durante o levantamento dos termos descritores do produto. Na ADQ é utilizada, via de regra, a escala não-estruturada de $9 \mathrm{~cm}$, ancorada em seus extremos com palavras que indicam a intensidade do atributo avaliado. Os resultados da ADQ são avaliados pela Análise de Variância (ANOVA) e Testes de Médias de Tukey, Análise Multivariada (Manova) 
e Análise de Componentes Principais (ACP), sendo efetuadas representações gráficas dos resultados (STONE e SIDEL, 1993). TASHIMA e CARDELLO (2003) utilizaram a Análise Descritiva Quantitativa para comparar o perfil sensorial de extrato hidrossolúvel de soja adoçado com sacarose e com sucralose. Verificaram que os atributos cor creme, aroma de baunilha, aroma de soja, sabor de baunilha, sabor de soja, doçura e viscosidade mostraram-se significativamente superiores para a amostra adoçada com sacarose.

Os métodos afetivos compreendem área muito útil para a análise sensorial, sendo realizados (geralmente) em seqüência aos testes discriminativos e descritivos. Esses métodos permitem medir o grau que os consumidores gostam ou desgostam de determinados produtos (testes de aceitação) e sua preferência entre produtos (testes de preferência).

O objetivo deste trabalho foi aplicar a Análise Descritiva Quantitativa para traçar o perfil sensorial de alimento com soja sabor laranja e comparar os resultados com as reações de potenciais consumidores de três produtos comerciais disponíveis no mercado.

\section{MATERIAL E MÉTODOS}

\subsection{MATERIAL}

Amostras de alimento com soja sabor laranja foram adquiridas no mercado, em embalagens longa vida de 1 litro e de $250 \mathrm{~mL}$, sendo duas da mesma marca (com adição de açúcar e light) identificadas respectivamente pelas letras $A$ e $B$ e outra de marca comercial identificada pela letra $C$. Os edulcorantes presentes na amostra B, segundo as especificações do rótulo, eram sucralose e acessulfame $\mathrm{K}$. Todos os testes foram realizados com os mesmos produtos.

Para os testes sensoriais, $20 \mathrm{~mL}$ de cada amostra foram servidas (a $15^{\circ} \mathrm{C}$ ) em copos plásticos descartáveis $(50 \mathrm{~mL})$ codificados com números de 3 dígitos. Os testes ocorreram no Laboratório de Análise Sensorial da Faculdade de Engenharia de Alimentos, Universidade de Campinas (UNICAMP), em cabines individuais. Os julgadores foram orientados a provar as amostras da esquerda para a direita. 


\subsection{ANÁLISE SENSORIAL}

\subsubsection{Análise Descritiva Quantitativa}

A terminologia descritiva e o perfil sensorial das amostras (A, B e C) foram desenvolvidos conforme STONE e SIDEL (1993).

\subsubsection{Pré-seleção dos julgadores}

Dez alunos de pós-graduação da UNICAMP, sem experiência prévia em análise descritiva (oito mulheres e dois homens), foram préselecionados com base na disponibilidade, interesse, facilidade de expressão e de trabalhar em grupo e habilidade para identificar atributos sensoriais.

\subsubsection{Desenvolvimento da terminologia descritiva}

Utilizou-se o Método de Rede de Kelly (MOSKOWITZ, 1983) para gerar os termos descritores que definem as similaridades e diferenças entre as amostras (Figura 1). Os julgadores discutiram os termos propostos, avaliando a freqüência com que apareciam e eliminaram os termos sinônimos ou pouco citados de forma consensual. Após definição dos termos descritores para a avaliação das amostras foi elaborada a Ficha de Avaliação das Amostras (Figura 2).

A equipe definiu de maneira consensual os materiais de referência que ajudaram na percepção de todas as características sensoriais do produto e para ancorar os extremos da escala de intensidade.

\subsubsection{Seleção e treinamento da equipe final}

Todos os membros da equipe sensorial passaram por treinamento. Foram utilizados os próprios produtos a serem avaliados e as amostras referência para fixar a memória sensorial, aguçar o poder de discriminação entre as amostras, avaliar a repetibilidade e a concordância com os demais membros da equipe (DAMÁSIO e COSTEL, 1991).

A apresentação das amostras foi monádica seqüencial, sendo solicitado que entre uma amostra e outra fosse enxaguado o palato com água (temperatura ambiente) e servida bolacha água e sal. 


\section{FIGURA 1 - MODELO DE FICHA EMPREGADA NO MÉTODO DE REDE DE KELLY}

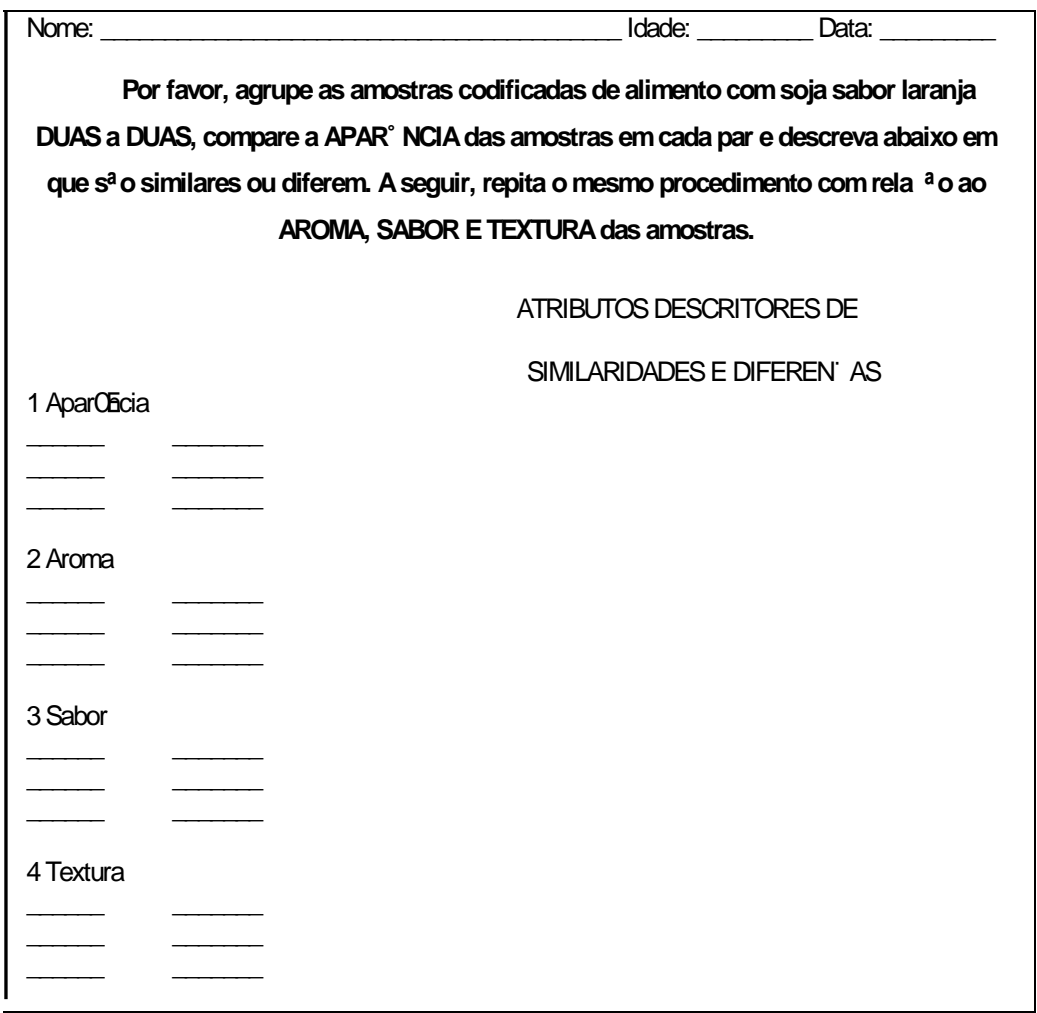

\subsubsection{Teste sensorial}

Os julgadores avaliaram as amostras com três repetições, utilizando a ficha definida para o teste (Figura 2). As amostras foram apresentadas de forma monádica seqüencial, em blocos completos casualizados, a fim de diminuir o efeito da ordem de apresentação das amostras. Os testes foram realizados em cabines individuais, sob luz branca.

\subsubsection{Análise dos resultados}

Utilizando o programa estatístico SAS (2001), os dados sensoriais foram submetidos a Análise de Variância (ANOVA) com duas fontes 
de variação (amostras e julgadores) e teste de comparação de médias de Tukey (significância de 5\%) e Análise de Componentes Principais (ACP).

\section{FIGURA 2 - MODELO DE FICHA EMPREGADA NA ANÁLISE DESCRITIVA QUANTITATIVA}

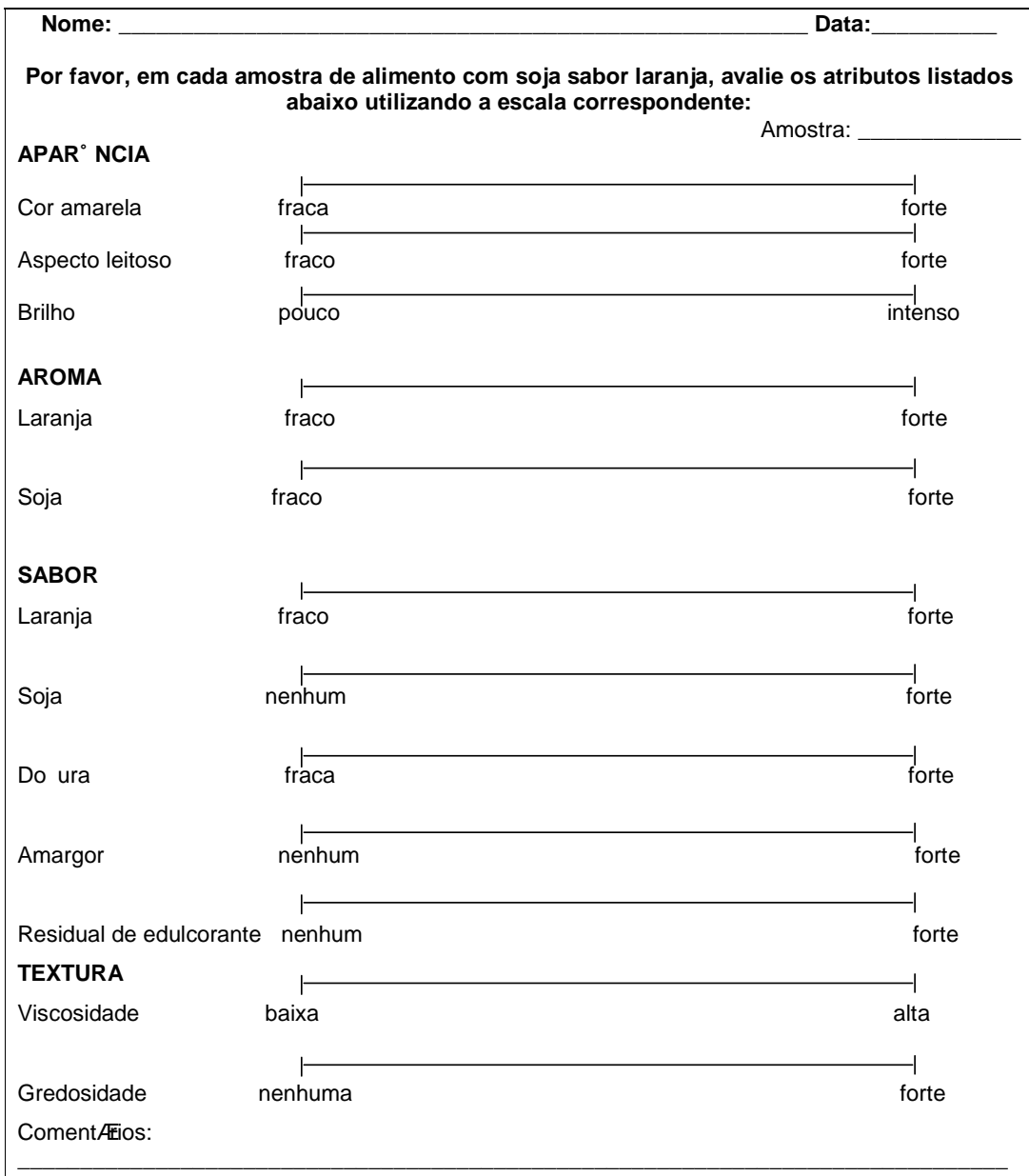




\subsubsection{Teste Afetivo}

A aceitação dos alimentos com soja sabor laranja por parte do público consumidor foi avaliada com a participação de 30 potenciais consumidores, pertencentes à comunidade da UNICAMP (alunos e funcionários).

As amostras foram apresentadas aos potenciais consumidores em blocos casualizados, de forma monádica seqüencial, utilizando-se escala hedônica estruturada de 9 pontos (QUEIROZ e GARCIA, 2000) (Figura 3). Os testes foram realizados em cabines individuais, sob luz branca.

\section{FIGURA 3 - MODELO DA FICHA EMPREGADA NO TESTE DE CONSUMIDOR UTILIZANDO ESCALA HEDÔNICA (ESTRUTURADA MISTA)}

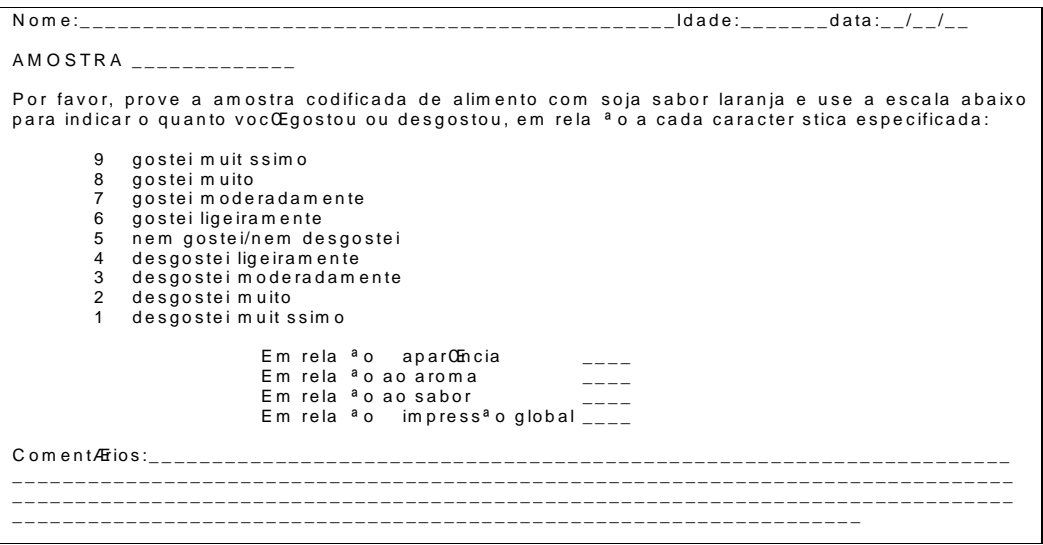

Os resultados foram avaliados pela Análise de Variância e pelo teste de médias de Tukey, utilizando-se o programa estatístico SAS (2001).

\section{RESULTADOS E DISCUSSÃO}

\subsection{ANÁLISE DESCRITIVA QUANTITATIVA}

Os 10 julgadores selecionados participaram das sessões do 
levantamento dos termos descritores, sendo escolhidos em consenso os atributos: coloração amarela, aspecto leitoso, brilho, aroma de laranja, aroma de soja, sabor de laranja, sabor de soja, sabor doce, sabor amargo, sabor residual de edulcorante, viscosidade e gredosidade (Tabela 1).

\section{TABELA1 - DEFINIÇÃO DOS TERMOS DESCRITORES DE ALIMENTO COM SOJA SABOR LARANJA E REFERÊNCIAS UTILIZADAS PELOS JULGADORES NOS TESTES DE ANÁLISE DESCRITIVA QUANTITATIVA}

\begin{tabular}{|c|c|c|c|}
\hline & $\begin{array}{l}\text { Termo } \\
\text { Descritor }\end{array}$ & Defini ${ }^{a} o$ & ReferC̄cias \\
\hline \multirow[t]{4}{*}{ AparC5cia } & Amarelo & $\begin{array}{l}\text { Intensidade da } \\
\text { cor amarela }\end{array}$ & 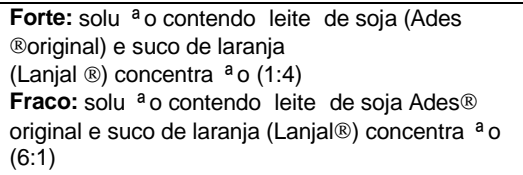 \\
\hline & Leitoso & AparCacia de leite & $\begin{array}{l}\text { Forte: leite de soja Ades }{ }^{\circledR} \text { original } \\
\text { Fraco: solu a o contendo leite de soja (Ades }{ }^{\circledR} \\
\text { original) e Aegua na propor a o de }(1: 10)\end{array}$ \\
\hline & Brilho & Intensidade de & Intenso: xarope de glicose (Karo®) \\
\hline & & reflex ${ }^{a}$ o da luz & $\begin{array}{l}\text { Pouco: Agua com farinha de trigo (Dona Benta }{ }^{\circledR} \text { ): } \\
0,33 \mathrm{~g} / \mathrm{mL}\end{array}$ \\
\hline \multirow[t]{3}{*}{ Aroma } & De laranja & Intensidade do & Forte: suco de laranja refrigerado Danone ${ }^{\circledR}$ \\
\hline & & aroma de laranja & $\begin{array}{l}\text { Fraco: solu }{ }^{a} \text { o contendo suco de laranja } \\
\text { refrigerado Danone }{ }^{\circledR} \text { e Agua na propor }{ }^{a} \text { o }(1: 16)\end{array}$ \\
\hline & De soja & $\begin{array}{l}\text { Intensidade do } \\
\text { aroma de soja }\end{array}$ & $\begin{array}{l}\text { Forte: solu a o contendo } 10 \mathrm{~g} \text { de extrato de soja } \\
\text { Mais Vita }{ }^{\circledR} \text { e } 100 \mathrm{~mL} \text { de Agua } \\
\text { Nenhum: Agua }\end{array}$ \\
\hline \multirow[t]{5}{*}{ Sabor } & De laranja & $\begin{array}{l}\text { Intensidade do } \\
\text { sabor de laranja }\end{array}$ & $\begin{array}{l}\text { Forte: suco de laranja refrigerado Danone }{ }^{\circledR} \\
\text { Fraco: solu a o contendo Ægua e suco de laranja } \\
\text { refrigerado Danone }{ }^{\circledR}(1: 8)\end{array}$ \\
\hline & De soja & $\begin{array}{l}\text { Intensidade do } \\
\text { sabor de soja }\end{array}$ & $\begin{array}{l}\text { Forte: solu a o de } 10 \mathrm{~g} \text { de extrato de soja Mais } \\
\text { Vita }{ }^{\circledR} \text { e } 100 \mathrm{~mL} \text { de Agua } \\
\text { Nenhum: Agua }\end{array}$ \\
\hline & Do ura & $\begin{array}{l}\text { Intensidade do } \\
\text { gosto doce }\end{array}$ & $\begin{array}{l}\text { Forte: solu a o de Agua com a œar ( } 30 \mathrm{~g} \text { de } \\
\text { a œear } / 200 \mathrm{~mL} \text { de Agua) } \\
\text { Fraco: solu a o de Agua a œear ( } 5 \mathrm{~g} \text { de a œear } \\
\text { /200 mL de Agua) }\end{array}$ \\
\hline & Amargor & $\begin{array}{l}\text { Intensidade do } \\
\text { gosto amargo }\end{array}$ & $\begin{array}{l}\text { Forte: solu a o de Agua com cafe na a } 0,02 \% \\
\text { Nenhum: Agua }\end{array}$ \\
\hline & $\begin{array}{l}\text { Residual de } \\
\text { edulcorante }\end{array}$ & $\begin{array}{l}\text { Intensidade do } \\
\text { gosto residual de } \\
\text { edulcorante }\end{array}$ & $\begin{array}{l}\text { Forte: solu a o de Agua com Adocyl }{ }^{\circledR} \text { ( } 5 \text { gotas / } \\
25 \mathrm{~mL} \text { de Agua) } \\
\text { Nenhum: Agua }\end{array}$ \\
\hline \multirow[t]{2}{*}{ Textura } & Viscosidade & Fluidez do I quido & 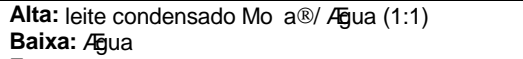 \\
\hline & Gredosidade & $\begin{array}{l}\text { Presen a de } \\
\text { part culas s lidas } \\
(p) \text { em } \\
\text { suspens }{ }^{a} \text { o }\end{array}$ & $\begin{array}{l}\text { Forte: solu a o de } 10 \mathrm{~g} \text { de extrato de soja Mais } \\
\text { Vita }{ }^{\circledR} \text { e } 100 \mathrm{~mL} \text { de Agua } \\
\text { Nenhum: Agua }\end{array}$ \\
\hline
\end{tabular}

${ }^{\circledR}=$ marca registrada. 
A Tabela 2 apresenta as médias de cada atributo avaliado para cada bebida ( $A, B$ e $C$ ). As amostras $A$ e $B$ não diferiram significativamente entre si ao nível de $5 \%$ em relação aos atributos aroma de laranja, aroma de soja, sabor de laranja, sabor de soja, doçura, amargor, viscosidade e gredosidade. Já a amostra $C$ não diferiu da amostra $B$ significativamente (ao nível de 5\%) em relação aos atributos doçura e amargor. As amostras A e C não diferiram significativamente (ao nível de $5 \%$ ) apenas nos atributos residual de edulcorante e viscosidade. Observou-se que as amostras A e B (produzidas pela mesma empresa) são as mais próximas entre si, diferindo apenas nos atributos cor amarela, aspecto leitoso, brilho e residual de edulcorante.

\section{TABELA 2 - RESULTADOS DO TESTE DE MÉDIAS DE TUKEY POR ATRIBUTO PARA AS AMOSTRAS DE ALIMENTO COM SOJA SABOR LARANJA}

\begin{tabular}{lccccc}
\hline Atributos & \multicolumn{3}{c}{ Mødia da Equipe Sensorial } & $F_{\text {amostra }^{*}}$ & MDS \\
& Amostra A & Amostra B & Amostra C & & \\
\hline Cor Amarela & $5,32^{\mathrm{b}}$ & $3,94^{\mathrm{c}}$ & $6,48^{\mathrm{a}}$ & 72,49 & 0,51 \\
Aspecto Leitoso & $3,58^{\mathrm{b}}$ & $6,18^{\mathrm{a}}$ & $1,90^{\mathrm{c}}$ & 145,47 & 0,61 \\
Brilho & $3,98^{\mathrm{b}}$ & $3,23^{\mathrm{c}}$ & $5,80^{\mathrm{a}}$ & 43,15 & 0,68 \\
Aroma de Laranja & $4,21^{\mathrm{a}}$ & $4,61^{\mathrm{a}}$ & $3,20^{\mathrm{b}}$ & 8,39 & 0,86 \\
Aroma de Soja & $2,10^{\mathrm{b}}$ & $2,29^{\mathrm{b}}$ & $4,34^{\mathrm{a}}$ & 46,62 & 0,62 \\
Sabor Laranja & $5,44^{\mathrm{a}}$ & $5,25^{\mathrm{a}}$ & $4,05^{\mathrm{b}}$ & 12,64 & 0,72 \\
Sabor Soja & $2,10^{\mathrm{b}}$ & $2,36^{\mathrm{b}}$ & $4,50^{\mathrm{a}}$ & 44,90 & 0,67 \\
Do ura & $5,21^{\mathrm{b}}$ & $5,58^{\mathrm{a}, \mathrm{b}}$ & $5,93^{\mathrm{a}}$ & 7,78 & 0,44 \\
Amargor & $1,08^{\mathrm{b}}$ & $1,72^{\mathrm{a}, \mathrm{b}}$ & $1,97^{\mathrm{a}}$ & 5,02 & 0,69 \\
Sabor residual de & & & & & \\
edulcorante & $0,65^{\mathrm{b}}$ & $3,75^{\mathrm{a}}$ & $0,69^{\mathrm{b}}$ & 77,16 & 0,69 \\
Viscosidade & $2,85^{\mathrm{a}, \mathrm{b}}$ & $3,01^{\mathrm{a}}$ & $2,44^{\mathrm{b}}$ & 3,47 & 0,54 \\
Gredosidade & $1,50^{\mathrm{b}}$ & $1,48^{\mathrm{b}}$ & $2,20^{\mathrm{a}}$ & 7,88 & 0,48 \\
\hline
\end{tabular}

MDS = Mínima Diferença Significativa pelo Teste de Tukey ao nível de 5\%.

${ }^{*} \mathrm{~F}$ de Snedecor. Todos os atributos apresentaram $\mathrm{F}$ amostra significativo ao nível de $5 \%$.

As médias das amostras acompanhadas pela mesma letra, na mesma linha, não diferem entre si pelo teste de Tukey.

O gráfico aranha sugere que as amostras A e B assemelham-se bastante, embora a A apresente cor amarela um pouco mais marcante, 
menos aspecto leitoso e amargor (Figura 4). A amostra B, caracterizada pelo aspecto leitoso, apresentou sabor residual de edulcorante bastante representativo, praticamente nulo nas demais amostras. A amostra C, com perfil bastante diferente das demais, apresentou sabor e aroma de soja acentuados, maior doçura, brilho, gredosidade e cor amarela.

\section{FIGURA 4 - REPRESENTAÇÃO GRÁFICA DOS RESULTADOS DA ANÁLISE DESCRITIVA QUANTITATIVA DE ALIMENTO COM SOJA SABOR LARANJA}

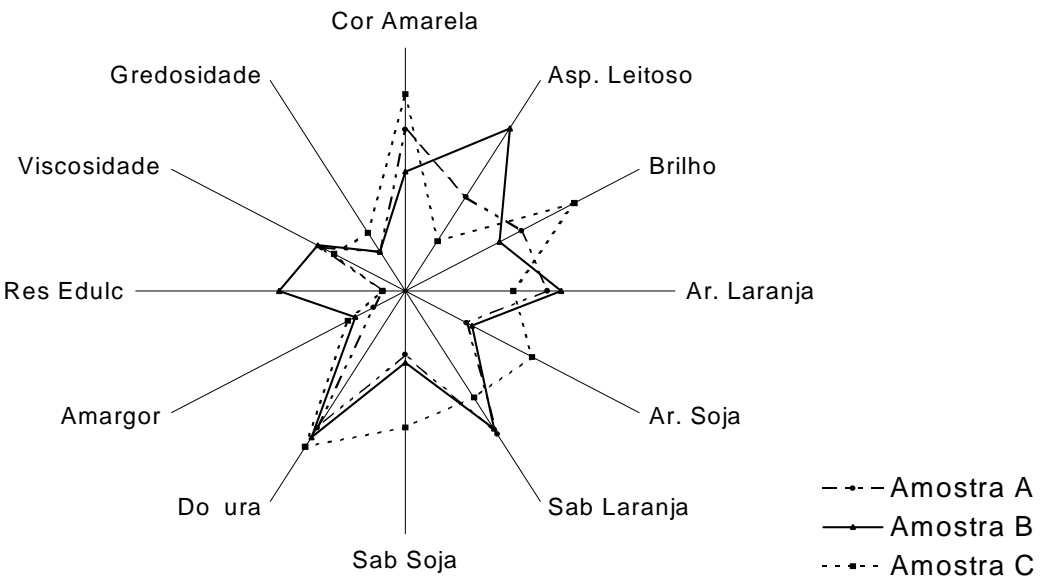

Asp. Leitoso = Aspecto Leitoso; Ar. Laranja = Aroma de Laranja; Ar. Soja = Aroma de Soja; Sab Laranja = Sabor de Laranja; Sab. Soja = Sabor de Soja; Res. Edulc $=$ Sabor Residual de Edulcorante.

A representação gráfica da Análise de Componentes Principais (ACP) está apresentada na Figura 5. A distinção entre as amostras pode ser observada pela localização bem definida de cada amostra no gráfico. Verificou-se que $69,46 \%$ da variação ocorrida entre as amostras foi explicada pelo primeiro eixo (Componente Principal 1) e 18,10\% pelo segundo (Componente Principal 2). Os Componentes Principais 1 e 2 explicaram juntos $87,56 \%$ da variação entre as amostras. 


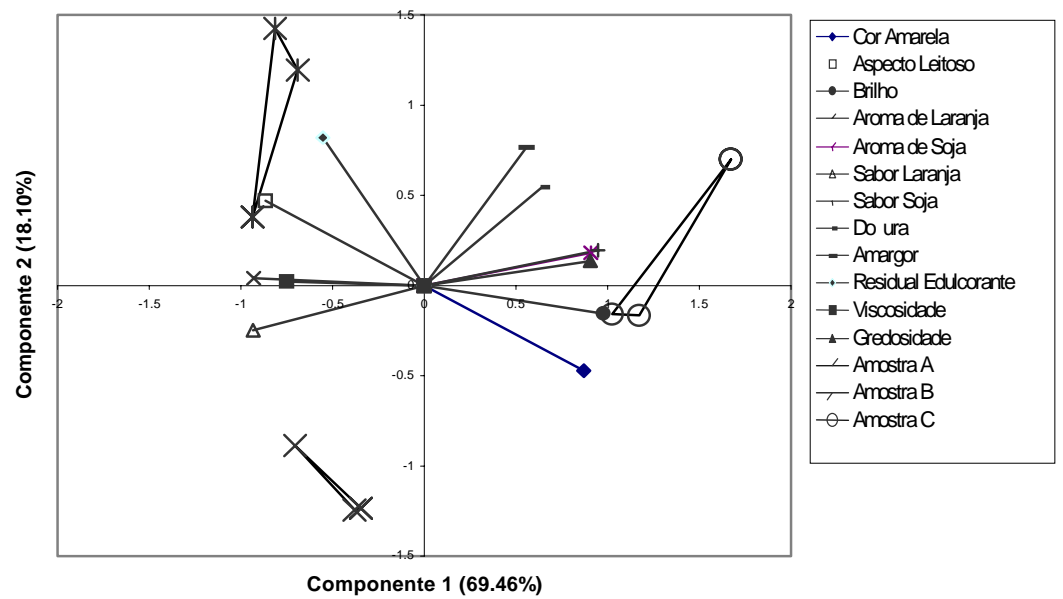

Os termos descritores que aparecem como vetores foram: cor amarela, aspecto leitoso, brilho, aroma de laranja, aroma de soja, sabor de laranja, sabor de soja, doçura, amargor, residual de edulcorante, viscosidade e gredosidade.

Quanto mais longo o vetor, melhor a explicação da variabilidade entre as amostras. Nesse caso, quase todos os atributos têm a mesma importância na identificação das amostras, já que quase todos são do mesmo tamanho. O vetor viscosidade (mais curto) sugere que as amostras não diferem muito com relação à viscosidade.

A amostra C caracterizou-se pelo sabor de soja, aroma de soja, gredosidade e brilho (Figura 5), sendo também marcantes a cor amarela, doçura e o amargor. A amostra B caracterizou-se pelo sabor residual de edulcorante e aspecto leitoso, sendo importantes também o aroma de laranja e a viscosidade. Já a posição da amostra A (pouco afastada dos vetores) sugere inexistência de características tão marcantes como as demais amostras. $O$ vetor mais representativo da amostra $A$ foi o sabor de laranja. 
Vetores próximos uns dos outros possivelmente apresentam alta correlação positiva entre si, como ocorreu com o sabor de soja, o aroma de soja e a gredosidade. Vetores paralelos em sentidos opostos representam correlação negativa (expresso na oposição cor amarela/ aspecto leitoso), ou seja, quanto mais leitoso, menos coloração amarela. A posição sabor de laranja/sabor de soja indica que o sabor de laranja pode ser inibido pelo forte sabor de soja.

\subsection{TESTE AFETIVO}

Os resultados dos testes de aceitação estão apresentados nas Figuras 6, 7, 8 e 9. A amostra A obteve maior concentração de notas nos valores mais elevados da escala para todos os atributos analisados. As notas atribuídas à amostra $B$ mostraram-se relativamente próximas à amostra $A$ em relação aos atributos aparência e aroma e inferiores em relação ao sabor e impressão global. A amostra $C$ revelou concentração de notas na parte inferior da escala (desgostei ligeiramente), principalmente em relação ao sabor.

\section{FIGURA 6 - DISTRIBUIÇÃO DAS NOTAS DOS JULGADORES - ATRIBUTO APARÊNCIA DE ALIMENTO COM SOJA SABOR LARANJA}

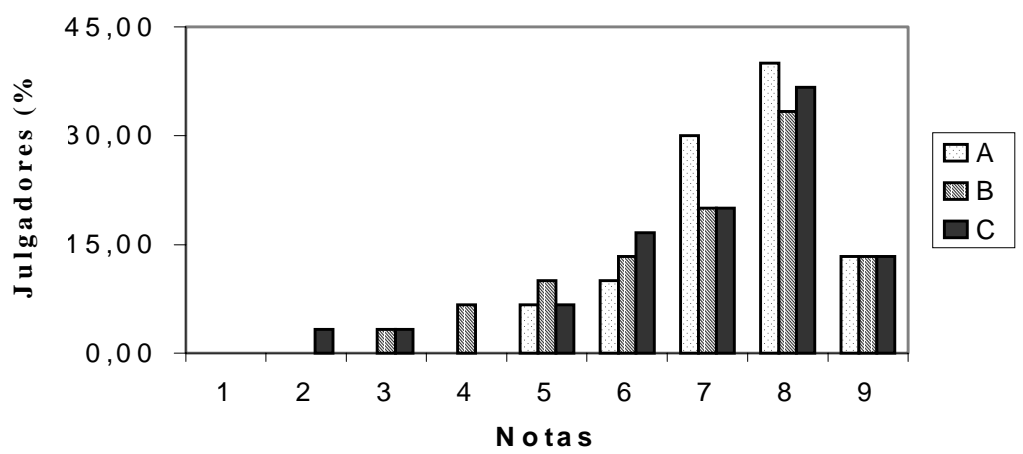

Não foi verificada diferença significativa entre as amostras em relação à aparência, ao nível de 5\% de significância (Tabela 3). Observou-se diferença significativa entre os julgamentos (Tabelas 3 e 4) em relação aos atributos aparência e aroma ao nível de $5 \%$ de significância. Os 
dados das Tabelas 4, 5 e 6 mostraram diferença significativa entre pelo menos uma das amostras para os atributos aroma, sabor e impressão global. As amostras $A$ e $B$ não diferiram significativamente entre si (ao nível de 5\%) em relação a todos os atributos analisados (Tabela 7).

\section{TABELA 3 - RESULTADOS DA ANÁLISE DE VARIÂNCIA (ANOVA) PARA O ATRIBUTO APARÊNCIA DE ALIMENTO COM SOJA SABOR LARANJA}

\begin{tabular}{lccccc}
\hline \multicolumn{1}{c}{ FV } & GL & SQ & SQM & $\mathbf{F}_{\text {calculado }}$ & $\mathbf{F}_{\text {tabelado }}$ \\
\hline Amostra & 2 & 4,20 & 2,10 & $1,20^{\text {ns }}$ & 3,16 \\
Julgador & 29 & 88,40 & 3,05 & $1,74^{*}$ & 1,71 \\
Res duo & 58 & 101,80 & 1,76 & & \\
Total & 89 & 194,40 & & & \\
\hline
\end{tabular}

* significativo ao nível de $5 \%$ de significância. ns = não significativo.

$\mathrm{FV}=$ Fonte de Variação; $\mathrm{GL}=$ Graus de Liberdade; $\mathrm{SQ}=$ Soma dos Quadrados; $\mathrm{SQM}=$ Soma dos Quadrados Médios.

\section{FIGURA 7 - DISTRIBUIÇÃO DAS NOTAS DOS JULGADORES - ATRIBUTO AROMA DE ALIMENTO COM SOJA SABOR LARANJA}

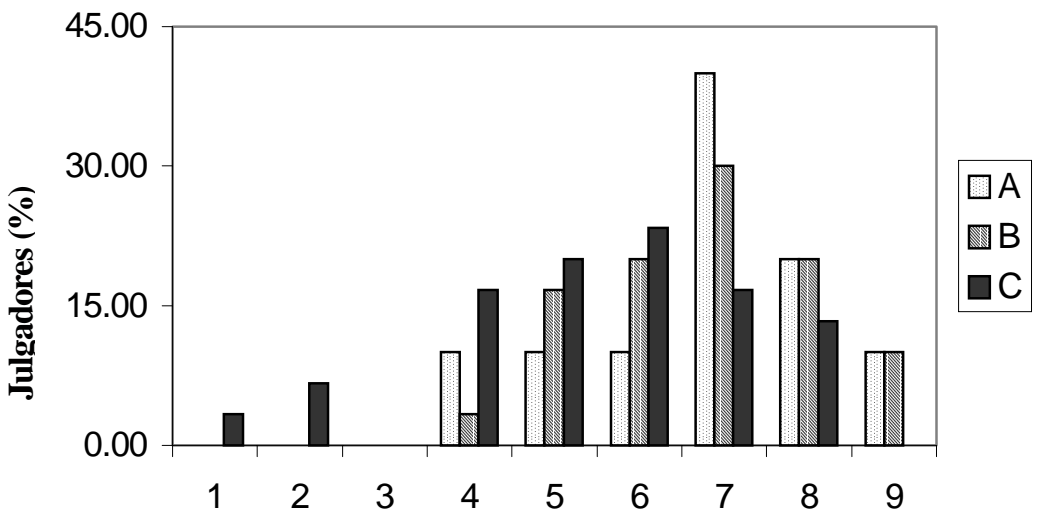

Notas 
TABELA 4 - RESULTADOS DA ANÁLISE DE VARIÂNCIA (ANOVA) PARA O ATRIBUTO AROMA DE ALIMENTO COM SOJA SABOR LARANJA

\begin{tabular}{lccccc}
\hline \multicolumn{1}{c}{ FV } & GL & SQ & SQM & $\mathbf{F}_{\text {calculado }}$ & $\mathbf{F}_{\text {tabelado }}$ \\
\hline Amostra & 2 & 34,69 & 17,35 & $10,71^{*}$ & 3,16 \\
Julgador & 29 & 111,66 & 3,85 & $2,38^{*}$ & 1,71 \\
Res duo & 58 & 93,97 & 1,62 & & \\
Total & 89 & 240,32 & & & \\
\hline
\end{tabular}

* significativo ao nível de $5 \%$ de significância.

FV = Fonte de Variação; GL = Graus de Liberdade; $S Q$ = Soma dos Quadrados; $\mathrm{SQM}=$ Soma dos Quadrados Médios.

\section{FIGURA 8 - DISTRIBUIÇÃO DAS NOTAS DOS JULGADORES - ATRIBUTO SABOR DE ALIMENTO COM SOJA SABOR LARANJA}

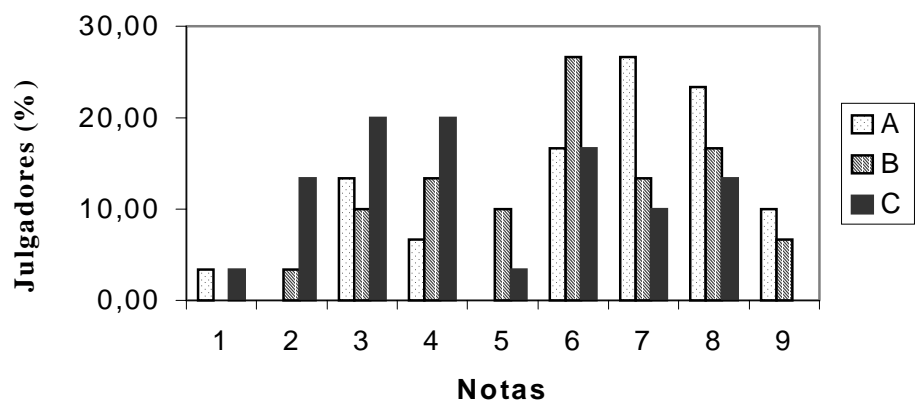

TABELA 5 - RESULTADOS DA ANÁLISE DE VARIÂNCIA (ANOVA) PARA O ATRIBUTO SABOR DE ALIMENTO COM SOJA SABOR LARANJA

\begin{tabular}{lccccc}
\hline \multicolumn{1}{c}{ FV } & GL & SQ & SQM & $\mathbf{F}_{\text {calculado }}$ & $\mathbf{F}_{\text {tabelado }}$ \\
\hline Amostra & 2 & 46,29 & 23,15 & $6,40^{*}$ & 3,16 \\
Julgador & 29 & 153,39 & 5,29 & $1,46^{\text {ns }}$ & 1,71 \\
Res duo & 58 & 209,71 & 3,62 & & \\
Total & 89 & 409,39 & & & \\
\hline
\end{tabular}

* significativo ao nível de $5 \%$ de significância.

$\mathrm{FV}=$ Fonte de Variação; $\mathrm{GL}=$ Graus de Liberdade; $\mathrm{SQ}=$ Soma dos Quadrados; $\mathrm{SQM}=$ Soma dos Quadrados Médios. 
FIGURA 9 - DISTRIBUIÇÃO DAS NOTAS DOS JULGADORES ATRIBUTO IMPRESSÃO GLOBAL DE ALIMENTO COM SOJA SABOR LARANJA

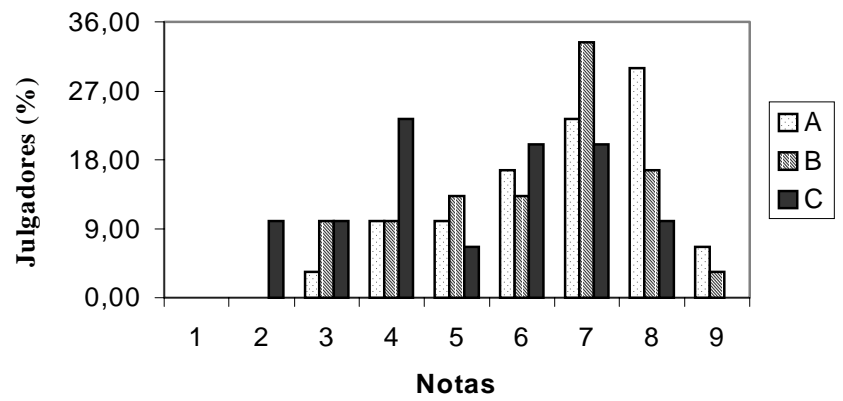

\section{TABELA 6 - RESULTADOS DA ANÁLISE DE VARIÂNCIA (ANOVA) PARA O ATRIBUTO IMPRESSÃO GLOBAL DE ALIMENTO COM SOJA SABOR LARANJA}

\begin{tabular}{lccccc}
\hline \multicolumn{1}{c}{ FV } & GL & SQ & SQM & $\mathbf{F}_{\text {calculado }}$ & $\mathbf{F}_{\text {tabelado }}$ \\
\hline Amostra & 2 & 33,36 & 16,68 & $5,87^{*}$ & 3,16 \\
Julgador & 29 & 91,96 & 3,17 & $1,17^{\text {ns }}$ & 1,71 \\
Res duo & 58 & 164,64 & 2,84 & & \\
Total & 89 & 289,96 & & & \\
\hline
\end{tabular}

* significativo ao nível de 5\% de significância.

$\mathrm{FV}=$ Fonte de Variação; $\mathrm{GL}=$ Graus de Liberdade; $\mathrm{SQ}=$ Soma dos Quadrados; $\mathrm{SQM}=$ Soma dos Quadrados Médios.

TABELA 7 - RESULTADOS DO TESTE DE MÉDIAS DE TUKEY POR ATRIBUTO PARA AS AMOSTRAS DE ALIMENTO COM SOJA SABOR LARANJA EM TESTE DE ACEITAÇÃO

\begin{tabular}{lccccc}
\hline Atributo & \multicolumn{3}{c}{ Mødia da Equipe Sensorial } & \multirow{2}{*}{$F_{\text {amostra }}$} & MDS \\
& Amostra A & Amostra B & Amostra C & & \\
\cline { 2 - 6 } AparCncia & $7,43^{\mathrm{a}}$ & $6,93^{\mathrm{a}}$ & $7,03^{\mathrm{a}}$ & 1,20 & 0,82 \\
\cline { 2 - 6 } Aroma & $6,80^{\mathrm{a}}$ & $6,77^{\mathrm{a}}$ & $5,47^{\mathrm{b}}$ & $10,70^{*}$ & 0,79 \\
\cline { 2 - 6 } Sabor & 6,33 & 5,87 & 4,63 & 6,40 & 1,18 \\
\cline { 2 - 6 } Impress & $6,6,13^{\mathrm{ab}}$ & $5,17^{\mathrm{b}}$ & $5,88^{*}$ & 1,04 \\
\hline
\end{tabular}

MDS = Mínima Diferença Significativa do Teste de Tukey ao nível de de 5\%.

${ }^{*}$ atributos que apresentaram $\mathrm{F}_{\text {amostra }}$ significativo ao nível de $5 \%$.

As médias das amostras acompanhadas pela mesma letra, na mesma linha, não diferem entre si pelo teste de Tukey. 


\section{CONCLUSÃO}

A Análise Descritiva Quantitativa evidenciou maior semelhança entre as amostras A e B, que diferiram apenas nos atributos cor amarela, aspecto leitoso, brilho e residual de edulcorante. Vale ressaltar que a formulação da amostra B está adequada em termos de doçura em relação a $A$, contudo o edulcorante utilizado deve ser alterado para diminuir o residual percebido pelos julgadores. Os demais atributos que diferiram entre as amostras $A$ e $B$ também poderiam ser aperfeiçoados na amostra $B$ no sentido de aproximá-la do produto tradicional. A amostra $\mathrm{C}$ diferiu da amostra $\mathrm{B}$ significativamente (ao nível de 5\%) em todos os atributos, com exceção da doçura e do amargor. As amostras A e $\mathrm{C}$ diferiram em todos os atributos (ao nível de $5 \%$ de significância), com exceção do residual de edulcorante e viscosidade.

O teste afetivo confirmou os resultados levantados pela Análise Descritiva Quantitativa, visto que a amostra A obteve as maiores médias e as maiores notas dos julgadores, não diferindo da amostra $B$ em nenhum dos aspectos analisados. A amostra $C$ diferiu da amostra $A$ em todos os atributos, mas não da amostra $B$ em relação à aparência e à impressão global.

\section{ABSTRACT}

\section{SENSORY PROFILE EVALUATION OF SOY FOOD PRODUCT WITH ORANGE FLAVOR}

Three commercial soy food products with orange flavor (A, B and C) conditioned in long life packing acquired in the market were sensory evaluated. Quantitative Descriptive Analysis (QDA) was used to trace the sensorial profiles evaluating 12 attributes raised through 10 selected and trained panelists. The acceptance of these products was evaluated by 30 potential consumers in a consumer test carried out in laboratory by utilizing mixed hedonic category of 9 points. The results of QDA was submitted to the Analysis of Variance (ANOVA), Tukey test of averages and Principal Components Analysis, although the results of the consumer test were analyzed by ANOVA and by the Tukey test of averages. The products $A$ and $B$ varied between each other and product $C$ differs from product $B$ significantly at $5 \%$ level in all attributes with the exception of sweetness and bitterness taste. The products $A$ and $C$ differed in all attributes with the exception to the artificial sweetener aftertaste and viscosity. In the Consumer test the product $A$ received 
the greater average scores of the panelists presenting no difference from product $B$ in none of the analyzed attributes.

KEY-WORDS: SOY PRODUCT; SENSORY ANALYSIS; QUANTITATIVE DESCRIPTIVE ANALYSIS; CONSUMER TEST.

\section{REFERÊNCIAS}

1 ABNT. Associação Brasileira de Normas Técnicas. NBR 12806: análise sensorial dos alimentos e bebidas. Rio de Janeiro, fev. 1993. 8 p.

2 ALIMENTOS funcionais. Food Ingredients, São Paulo, n. 9, p. 26-42, nov/ dez. 2000.

BEBIDAS: de sucos prontos, resfrescos, chá a bebidas energéticas, um bom negócio. Food Ingredients, São Paulo, n. 11, p. 34-42, mar/abr. 2001.

4 CIVILLE, G. V.; SZCESNIAK, A. S. Guideline to training a texture profile panel. Journal Texture Studies, v. 4, n. 2, p. 204-223, 1973.

5 DAMÁSIO, M. H.; COSTELL, E. Análisis sensorial descriptivo: generación de descriptores y selección de catadores. Revista de Agroquímica y Tecnologia de Alimentos, v.31, n. 2, p. 165-178, 1991.

KRONES. Sucos: consumo da bebida à base de soja aumenta no Brasil. Boletim Eletrônico da Krones S/A, n.6, setembro de 2002. Disponível em: <http://www.krones.com.br/Boletim/Edicao6/materia6.htm>. Acesso em: 05/09/2003.

MEILGAARD, M.; CIVILLE, G.V.; CARR, B.T. Sensory evaluation techniques. $3^{\text {rd }}$. New York: CRC, 1999. 281 p.

MOSKOWITZ, H. R. Product testing and sensory evaluation of foods. Westport: Food e Nutrition Press, 1983. 605 p.

9 NOTÍCIAS EMBRAPA SOJA. Evento revela panorama atual e perspectiva da soja no mundo. Disponível em: <http://www.cnpso.embrapa. br/noticia/ ver_noticia.php?cod_noticia=28>. Acesso em 05/02/2004.

10 QUEIROZ, M. B.; GARCIA, N. H. P. Avaliação do perfil sensorial de amêndoas de cupuaçu e cacau torradas utilizando análise descritiva quantitativa. Boletim do CEPPA, Curitiba, v. 18, n. 2, p. 249-266, 2000.

11 SAS INSTITUTE. Statistical analysis system, versão 8.0. Cary, N.C., 2001.

12 STONE, H.; SIDEL, J. L. Sensory evaluation practices. $2^{\text {nd }}$ ed. London: 
Academic Press. 1993. 337 p.

13 TASHIMA, E. H.; CARDELLO, H. M. A. B. Perfil sensorial de extrato hidrossolúvel de soja (Glicina Max L. Merril) comercial adoçado com sacarose e com sucralose. Boletim do CEPPA, Curitiba, v. 21, n. 2, p. 409-428, 2003. 